\title{
Review Article \\ The Role of Rhizobial ACC Deaminase in the Nodulation Process of Leguminous Plants
}

\author{
Francisco X. Nascimento, ${ }^{1}$ Clarisse Brígido, ${ }^{2,3}$ Bernard R. Glick, ${ }^{4}$ and Márcio J. Rossi ${ }^{1}$ \\ ${ }^{1}$ Laboratório de Bioprocessos, Departamento de Microbiologia, Universidade Federal de Santa Catarina, \\ 88040-900 Florianópolis, SC, Brazil \\ ${ }^{2}$ Instituto de Ciências Agrárias e Ambientais Mediterrânicas (ICAAM) (Laboratório de Microbiologia do Solo), \\ Universidade de Évora, Núcleo da Mitra, 7000-083 Évora, Portugal \\ ${ }^{3}$ Instituto de Investigação e Formação Avançada (IIFA), Universidade de Évora, 7000-083 Évora, Portugal \\ ${ }^{4}$ Department of Biology, University of Waterloo, Waterloo, ON, Canada N2L $3 G 1$ \\ Correspondence should be addressed to Francisco X. Nascimento; fxnascimento@gmail.com
}

Received 27 November 2015; Accepted 28 April 2016

Academic Editor: Othmane Merah

Copyright (c) 2016 Francisco X. Nascimento et al. This is an open access article distributed under the Creative Commons Attribution License, which permits unrestricted use, distribution, and reproduction in any medium, provided the original work is properly cited.

\begin{abstract}
Symbiotic rhizobia-legumes associations are extremely important in terms of sustainable agricultural practices. This symbiosis involves a complex interaction between both partners, plant and bacterium, for bacterial infection and the formation of symbiotic $\mathrm{N}$-fixing nodules. In this regard, the phytohormone ethylene plays a significant role in nodule formation, acting as an inhibitor of the nodulation process. Ethylene not only regulates nodule development but also regulates many other plant developmental cues, including various stress responses that inhibit overall plant growth. Some rhizobia produce the enzyme 1-aminocyclopropane-1carboxylate (ACC) deaminase, thus, being able to decrease ACC and, consequently, decrease deleterious ethylene levels that affect the nodulation process. This occurs because ACC is the immediate precursor of ethylene in all higher plants. Hence, rhizobia that express this enzyme have an increased symbiotic potential. In addition to the direct role that ACC deaminase plays in the nodulation process per se, in a limited number of instances, ACC deaminase can also modulate nodule persistence. This review focuses on the important role of rhizobial ACC deaminase during the nodulation process, emphasizing its significance to legume growth promotion.
\end{abstract}

\section{Introduction}

The symbiotic rhizobia-legume association is one of the most studied beneficial plant-microbe interactions. This symbiotic association has traditionally been used in agricultural practices to provide nitrogen to plants and, thereby, enhance plant growth [1]. This symbiosis involves legumes and a specific group of soil bacteria, collectively known as rhizobia, which are able to form root nodules and fix atmospheric $\mathrm{N}$ when associated with legumes. For the most part, the interest in rhizobia strains is a consequence of their ability to efficiently fix atmospheric nitrogen, making them an important component of sustainable agricultural practices.

The successful interaction between a legume and rhizobia, requires two main developmental processes for the formation of symbiotic nitrogen-fixing nodules: bacterial infection and nodule organogenesis $[2,3]$, which must be coordinated in both a spatial and a temporal manner in order to ensure nodule formation at the site of bacterial infection on the roots [4]. The plant-bacteria symbiosis is initiated by a complex signaling dialogue between legumes and compatible rhizobia, eventually allowing the entry of rhizobia into the root. Briefly, rhizobia form an intimate symbiotic relationship with legumes by responding chemotactically to various flavonoid molecules released by the legume host. These flavonoids bind specifically and tightly to the rhizobial NodD protein, which is the major determinant of rhizobial host specificity. Each strain of rhizobia recognizes only a limited number of flavonoid structures and each species of legumes 
produces its own specific set of flavonoids. The flavonoidNodD complex binds to a nodulation promoter element and induces the expression of the rhizobial nodulation genes, which in turn produces a lipochitooligosaccharide nod factor that binds to a legume root receptor and triggers mitotic cell division in roots, leading to nodule formation [3]. The development of nodules, which are plant organs wherein rhizobia reduce atmospheric nitrogen into ammonia [4], begins when the infection thread reaches the nodule primordium located in the root cortex that ultimately develops into a nodule upon release of the rhizobia [5] and after the bacteria differentiate into a specialized symbiotic organelle-like form, termed bacteroid. The process of symbiotic $\mathrm{N}_{2}$-fixation involves a large number of rhizobial genes, namely, the nif genes that encode the nitrogenase enzyme and its iron-molybdenum cofactor [6]. Unfortunately, many of the components of this symbiotic process, such as molecular signaling, rhizobial attachment, root hair curling, and infection thread formation, as well as the nodule formation and nitrogen fixation, are severely affected by various stresses [7-14]. In addition, rhizobia also have to deal with adverse conditions within the host cells, as well as with the plant's innate immunity/response, all of which may interfere with the symbiosis [15]. Nevertheless, rhizobia possess multiple mechanisms to counteract some of the negative effects associated with environmental stresses thereby optimizing the legume-rhizobia interaction during the nodulation process. One important strategy to counteract many of the effects of stress during the nodulation process used by rhizobia is the modulation of ethylene levels that negatively affect the nodulation process. Modulation of ethylene levels may occur either by the bacterium synthesizing rhizobitoxine [16] a competitive inhibitor of the plant enzyme 1-aminocyclopropane-1-carboxylate (ACC) synthase (the enzyme responsible for the synthesis of ACC) or by expression of the enzyme ACC deaminase which cleaves plant ACC into ammonia and $\alpha$-ketobutyrate [17]. Both mechanisms are able to decrease the ethylene levels in plant root tissue, at least locally in and around the root nodules, and have beneficial effects on the symbiotic rhizobium-legume process as well as on plant growth itself. In this work we discuss the role of ACC deaminase in the nodulation process of rhizobia and its effects in legume growth promotion.

\section{The Phytohormone Ethylene}

Ethylene is a gaseous plant hormone produced endogenously by all higher plants and is recognized as one of the most important molecules regulating plant growth and development $[18,19]$. This phytohormone regulates many plant developmental processes such as germination, root and shoot elongation, abscission, senescence, flowering, and fruit ripening as well as the responses to biotic and abiotic stress [19-23]. Many of the inhibitory effects of ethylene on plant growth occur as a consequence of stressful conditions. Under these conditions, the stressed plant first produces a small peak of ethylene that activates the transcription of various plant defensive genes [24]. Subsequently, the endogenous production of ethylene is substantially accelerated and generates a second and much greater peak of ethylene, which adversely affects plant growth, often turning on the transcription of genes associated with plant senescence [21]. Some of the effects of various stresses on plants are not solely attributed to the stress itself but rather are due to the autocatalytic ethylene synthesis that ensues following the stress. Therefore, for optimal growth and development, regulation of ethylene production in plant tissues is essential $[25,26]$.

Ethylene biosynthesis in plants occurs via a methionine dependent pathway, which was firstly described by Adams and Yang [27]. In this biosynthetic pathway, methionine is converted to S-adenosyl methionine (SAM) by the enzyme SAM synthase. SAM is then converted to ACC, the immediate ethylene precursor, by the action of the enzyme ACC synthase. Ultimately, ACC is converted to ethylene by the ACC oxidase enzyme. The limiting step in the plant ethylene biosynthetic pathway is the conversion of SAM to ACC by the enzyme ACC synthase, indicating the key role of ACC in plant ethylene production [28]. Nevertheless, there are some reports of limitation of ethylene formation. For instance, during hypoxia ACC cannot be converted to ethylene by ACC oxidase in the absence of oxygen $[29,30]$.

\section{Ethylene Effects in the Nodulation Process}

In leguminous plants, ethylene is known for its negative role in the nodulation process initiated by rhizobia, as it inhibits the formation and functioning of nodules [31-33]. The first study of the effect of ethylene on legume nodulation was reported by Grobbelaar et al. [34]. These authors showed that exogenous ethylene $(0.4 \mathrm{ppm})$ greatly inhibited the nodulation process of Phaseolus vulgaris. Similarly, Drennan and Norton [35] demonstrated that the application of ethephon, a liquid ethylene-releasing compound, reduced nodule number in Pisum sativum. Later, Goodlass and Smith [36] confirmed these results, showing that exogenous ethylene application reduced the number of nodules formed and also the nitrogen fixation abilities, in P. sativum and Trifolium repens. Application of the ACC synthase inhibitor AVG (aminoethoxyvinylglycine) induced an increase in nodule formation in Medicago sativa plants [37]. Similarly, Fearn and LaRue [38] showed that the application of ethylene biosynthesis and perception inhibitors restored the nodulation profile of sym 5 pea plants which were found to be more sensitive to normal levels of ethylene. In a more detailed study, Lee and LaRue [39] showed that exogenous ethylene application inhibited nodulation on the primary and lateral roots of P. sativum L. cv Sparkle and Melilotus alba. These authors also showed that treating the roots with $1 \mu \mathrm{M}$ $\mathrm{Ag}+$, an ethylene perception blocker, diminished the ethylene inhibitory effects. Ethylene biosynthesis inhibitors, AVG and AOA (aminooxyacetic acid), increased the nodulation, while ethephon reduced the number of nodules formed in $P$. vulgaris [40]. Although not all legumes respond similarly, addition of exogenous ethylene to most nodulating plants reduces the frequency of nodule primordia formation [41, 42].

In addition, experiments with mutant/transgenic plants have also contributed to the understanding of the role of ethylene in the regulation of the nodulation process. In this 
instance, Penmetsa and Cook [43] showed that Medicago truncatula sickle mutants, insensitive to ethylene, formed an increased number of nodules compared to the wild-type form of this plant. Later, Penmetsa et al. [44] identified sickle mutants as being defective in a gene homologous to the Arabidopsis EIN2 (ethylene insensitive 2) gene. In agreement, the silencing of two Lotus japonicus EIN2 homologous genes also resulted in increased nodule formation [45]. In a study performed by Nukui et al. [46], transgenic L. japonicus plants expressing the mutant melon ethylene receptor gene Cm-ERS1/H70A inoculated with Mesorhizobium loti showed markedly higher numbers of infection threads and nodule primordia, suggesting that ethylene perception assists the negative feedback regulation of secondary nodule initiation. Likewise, the expression of the Arabidopsis etr1-1 (a mutant ethylene receptor) gene in $L$. japonicus also leads to an increased infection thread formation [47]. Therefore, ethylene is also involved in the development of infection threads, especially infection thread initiation and elongation [2].

Several studies have shown that ethylene can inhibit numerous steps of the nodulation process (reviewed by Ferguson and Mathesius [48]). In this sense, ethylene may be involved in several phases of symbiosis, including the initial response to bacterial Nod factors, nodule development, senescence, and abscission [49, 50]. Oldroyd et al. [42] suggested that ethylene inhibits the calcium spiking process responsible for the perception of bacterial Nod factors in Medicago truncatula. The work of Lee and LaRue [39] indicated that exogenous ethylene did not lead to a decrease in the number of infections, but rather nearly all of the infections were blocked when the infection thread was in the basal epidermal cell or in the outer cortical cells of the plant. This leads to a reduction in infection as well as in the number of nodules in legumes. Heidstra et al. [51] postulated that a gradient of ethylene is responsible for restricting nodules radially to positions opposite to the xylem poles. Moreover, ethylene controls the epidermal responses during the nodulation process and, thus, negatively regulates multiple epidermal responses in order to inhibit rhizobial infection $[46,52]$. Curiously, several studies have shown that radial restriction of nodule positioning is not observed in ethylene insensitive mutants [43, 47, 53], further confirming the hypothesis. Additionally, it was shown that endogenous ethylene production significantly increases in roots infected by Rhizobium or Bradyrhizobium, consequently decreasing the number of nodules that form on the infected plants $[54,55]$.

Recent studies show an intricate web of molecular mechanism underlying the plant control over nodulation. In this sense, ethylene acts as a major participant in the autoregulation of nodulation $(\mathrm{AON})$ process $[2,56,57]$.

\section{Mechanisms Employed by Rhizobia to Modulate Plant Ethylene Levels}

It is essential to regulate the ethylene level in the plant roots in order to achieve an improved symbiotic association [47]. As mentioned above, rhizobia have mechanisms that help to modulate the ethylene levels in plant roots; these include the bacterial enzyme ACC deaminase and the synthesis of the molecule rhizobitoxine.

The rhizobial enzyme ACC deaminase cleaves ACC (the immediate precursor of ethylene in plants) to ammonia and $\alpha$-ketobutyrate, both of which are readily metabolized by the bacterium or the plant [26]. ACC deaminase-producing rhizobial cells can reduce ethylene concentrations in the infection threads and increase the persistence of infection threads by suppressing the defense signals in the plant cells, thus increasing the extent of nodulation of legume roots [5862].

On the other hand, rhizobitoxine, an enol-ether amino acid, reduces the ethylene levels in plant roots in two different ways: (i) it inhibits the enzyme $\beta$-cystathionase which is necessary for methionine biosynthesis [52] and (ii) it inhibits the enzyme ACC synthase in the ethylene biosynthesis pathway $[16,52]$. Rhizobitoxine-possessing strains have been found to be highly effective in enhancing nodulation and competitiveness in Amphicarpaea edgeworthii and Vigna radiata [63] and in Macroptilium atropurpureum [16]. In addition, rhizobitoxine is beneficial to rhizobia living inside nodules by allowing more rhizobial reproduction or by enhancing the synthesis of poly-3-hydroxybutyrate to support lateral reproduction $[64,65]$. Despite, the positive effect of rhizobitoxine in the nodulation process, its production appears to be limited to very few rhizobial species (mainly Bradyrhizobium). On the other hand, ACC deaminase genes are found in a wide range of different rhizobial species [66].

Ultimately, either ACC deaminase or rhizobitoxine production may be helpful in the nodulation process and thereby increase the nitrogen supply for legume plants due to a more effective nodulation. This may be especially important when plants are growing under stressful conditions so that ethylene may attain levels that are highly inhibitory to nodulation.

\section{ACC Deaminase in Rhizobia}

ACC deaminase (encoded by acdS genes) are prevalent in many rhizobial species, including $\alpha$ and $\beta$-rhizobia [66], although it is important to note that not all strains within a particular species contain this enzyme. In $\alpha$-rhizobia, acdS genes are found in bacteria such as Azorhizobium, Bradyrhizobium, Methylobacterium, Mesorhizobium, Rhizobium, and Ensifer (Sinorhizobium), Devosia, Microvirga, and Bosea. In $\beta$-rhizobia, acdS genes are found in Burkholderia and Cupriavidus.

Ma et al. [67] and later Duan et al. [68] demonstrated ACC deaminase activity in several Rhizobium spp. (R. leguminosarum, R. gallicum, and R. hedysari). Sinorhizobium meliloti SM11, carrying the acdS gene in a plasmid, is able to produce ACC deaminase [69]. The Mesorhizobium sp. MAFF303099 acdS gene encodes a functional ACC deaminase [70]. Bradyrhizobium japonicum is also able to degrade ACC through ACC deaminase production [71]. Recently, Fedorov et al. [72] have characterized Methylobacterium nodulans ACC deaminase enzyme. ACC deaminase activity has also been demonstrated in members of $\beta$-rhizobia, 
Burkholderia phymatum STM815, and Burkholderia tuberum STM678 [73].

Despite the ability to breakdown ACC and use it as a nitrogen source, many of these strains presented different levels of ACC deaminase activity under free-living conditions. For instance, Duan and colleagues [68] showed that ACC deaminase activity ranged from 0.076 to $0.274 \mu \mathrm{mol} \alpha$ ketobutyrate/mg protein/h in Rhizobium spp. Stiens et al. [69] and Kong et al. [74] reported a value of 0.355 and $0.180 \mu \mathrm{mol}$ $\alpha$-ketobutyrate/mg protein/h for S. meliloti SM11 and $S$. meliloti CCNWSX0020 ACC deaminase activity, respectively. No ACC deaminase activity was detected in Mesorhizobium sp. MAFF303099 under free-living conditions; however, in a bacteroid state (inside the formed nodules), Mesorhizobium sp. MAFF303099 showed an ACC deaminase activity of approximately $0.075 \mu \mathrm{mol} \alpha$-ketobutyrate $/ \mathrm{mg}$ protein $/ \mathrm{h}$ [70]. On the other hand, bacterium like Bradyrhizobium japonicum presented a free-living ACC deaminase activity of $1.49 \mu \mathrm{mol} \alpha$-ketobutyrate/mg protein/h [71], whereas Burkholderia strains showed higher ACC deaminase activities, ranging from 3.55 to $4.63 \mu \mathrm{mol} \alpha$-ketobutyrate $/ \mathrm{mg}$ protein/h [73]. Although different methodologies and study conditions may account for some discrepancies between the ACC deaminase activity values, it is possible that these strains (even with similar acdS gene sequences) present different ACC deaminase activities due to factors, such as, acdS location in the replicon, acdS copy number, and acdS gene transcriptional regulation. Nevertheless, rhizobia typically exhibit only a low level of enzyme activity compared with free-living plant growth-promoting bacteria (i.e., 10- to 100fold less than free-living bacteria), suggesting the possibility that there may be at least two types of ACC deaminaseproducing bacteria [75]. There are free-living bacteria that bind relatively nonspecifically to plant roots and have a high level of ACC deaminase activity, protecting plants from different stresses by lowering ethylene levels throughout the plant. Alternatively, rhizobia bind tightly to the roots of specific plants and have a low level of enzyme activity that facilitates nodulation by locally lowering ethylene levels [26].

In Rhizobium and Sinorhizobium strains, acdS genes are mostly found in symbiotic plasmids, while Azorhizobium, Bradyrhizobium, and Mesorhizobium typically possess acdS genes in the chromosome. Still, in most Mesorhizobium spp. acd $S$ genes are located in symbiotic islands next to the symbiotic genes [76]. This is not observed in Azorhizobium or Bradyrhizobium where acdS genes are found in chromosomal locations far away from the symbiotic island [66]. In contrast, root-nodulating Burkholderia and Cupriavidus strains not only possess an acdS gene in a second chromosome, but also possess a second acdS gene copy in a symbiotic plasmid [66]. In this regard, horizontal gene transfer has been proposed for the acquisition of ACC deaminase genes by some rhizobia $[66,76]$.

Despite the presence of acdS genes in the genomes of a number of rhizobia strains, not much is understood about their functionality and role in the nodulation process. To date, some studies have reported ACC deaminase activity in some of these strains, but not all strains display enzyme activity when this activity is induced by ACC under freeliving conditions, suggesting diverse types of regulation or the requirement for different elements for induction. In this sense, two different modes of regulation of the acdS gene have been identified. For instance, studies performed by $\mathrm{Ma}$ et al. [67] showed that a gene encoding an LRP-like protein (termed $a c d R$ ) controls $R$. leguminosarum acdS transcription. $R$. leguminosarum $a c d R$ gene deletion resulted in a loss of ACC deaminase activity [67]. Analysis of completely sequenced bacterial genomes showed that $a c d R$ is found in most bacterial strains possessing an acdS gene (including Azorhizobium, Bradyrhizobium, Methylobacterium, Rhizobium, Sinorhizobium, Burkholderia, and Cupriavidus) consistent with the conclusion that $a c d R$ is a common regulator of acdS gene transcription [66]. In addition, analysis of acdS and $a c d R$ gene sequences showed that $a c d R$ is more prone to divergence than acdS, and this fact may, in part, account for a fine-tuning of acdS expression [66]. Curiously, most Mesorhizobium strains do not possess acdR genes [66]. In most Mesorhizobium spp. studied acdS genes are found in a chromosomal symbiotic island and are only expressed in symbiotic conditions under transcriptional control of the NifA protein $[70,76,77]$. Analysis of the upstream regions of the acdS gene in many Mesorhizobium spp. indicate a putative NifA binding site, suggesting that NifA regulation of acdS expression may be common within the Mesorhizobium genus [76]. This is consistent with the fact that most Mesorhizobium strains do not produce ACC deaminase activity under free-living conditions [58, 77-79]. However, Safronova et al. [80] detected free-living ACC deaminase activity in two Mesorhizobium loti strains. It is possible that in some Mesorhizobium strains the acdS gene is not present in a chromosomal symbiotic island under transcriptional control of NifA, but rather it is located in a symbiotic plasmid under transcriptional control of $a c d R$ (as found in Rhizobium or Sinorhizobium strains). This is consistent with the presence of acdS and $a c d R$ in $M$. alhagi CCNWXJ12-2 [66]. This fact may account for the free-living ACC deaminase activity of some Mesorhizobia.

\section{ACC Deaminase Effects in the Nodulation Abilities of Rhizobia}

Studies using rhizobial acdS deletion mutants as well as rhizobial strains expressing exogenous acdS genes have shown the important role of ACC deaminase in the nodulation process. For instance, Ma and coworkers [67] reported, for the first time, the effect of ACC deaminase gene in the nodulation process. Thus, an acdS minus mutant of $R$. leguminosarum bv. viciae $128 \mathrm{C} 53 \mathrm{~K}$ showed a $25 \%$ reduction of its nodulation abilities in P. sativum cv. Sparkle. In addition, a 23\% decrease in shoot dry weight was observed in plants inoculated with the acdS mutant strain. The authors also showed that ACC deaminase activity did not have any influence on nitrogenase activity inside the formed nodules, further suggesting that ACC deaminase is involved only in the early stages of nodule development (infection thread formation) but not in nodule function (nitrogen fixation) per se. Uchiumi et al. 
[70] showed that a Mesorhizobium sp. MAFF303099 acdS mutant had decreased symbiotic abilities. The acdS mutant strain formed fewer nodules in L. japonicus than its wild-type counterpart and also showed decreased nodule occupancy abilities. In both of the above mentioned cases, the ACC deaminase gene knockout resulted in a decreased ability to nodulate its host plant when compared to its respective wildtype strain, indicating that the presence of such gene plays an important role in the symbiotic efficiency and increases legume nodulation. On the other hand, rhizobial expression of exogenous ACC deaminase genes results in the increase of both nodulation efficiency and rhizobial competiveness. In this regard, Ma et al. [59] observed that S. meliloti Rm1021 that had been transformed to express an exogenous ACC deaminase gene had an increased ability to nodulate $M$. sativa plants. By expressing the acdS and $a c d R$ gene from $R$. leguminosarum bv. viciae $128 \mathrm{C} 53 \mathrm{~K}$, S. meliloti Rm1021 was able to produce 35 to $40 \%$ more nodules when compared to its wild-type form. The transformed strain also had an increased competitiveness in colonizing the nodules formed in $M$. sativa plants. Ma and coworkers [67] postulated that the transformants ability to utilize ACC as an extra nutrient source could make the bacterium proliferate better in the infection threads when compared to those that do not express ACC deaminase. Therefore, infecting cells that produce ACC deaminase are more likely to reach nodule primordia and form mature nodules. Similarly, Mesorhizobium sp. MAFF303099 expressing ACC deaminase under freeliving conditions presented increased nodulation efficiency and competitiveness [79]. These authors integrated an extra copy of the Mesorhizobium. sp MAFF303099 acdS gene into the Mesorhizobium sp. MAFF303099 chromosome under the transcriptional control of a constitutive promoter. The acdS-transformed strain induced the formation of a higher number of nodules and was more competitive than the wildtype strain on L. japonicus and L. tenuis plants. Nascimento et al. [78] also showed that Mesorhizobium ciceri LMS-1 expressing an exogenous ACC deaminase was able to form an increased number of nodules in Cicer arietinum plants. In this instance, the plasmid pRKACC containing the acdS and $a c d R$ genes of Pseudomonas sp. UW4 was inserted and maintained in M. ciceri LMS-1, which resulted in free-living ACC deaminase activity. By expressing an exogenous ACC deaminase the pRKACC-transformed strain enhanced its nodulation activity by $127 \%$ when compared to the wildtype strain and consequently increased chickpea biomass by $125 \%$. Nodulation assays showed that the pRKACCtransformed strain was able to form more developed nodules in earlier stages of nodulation (31 days after inoculationDAI); however, it was only at later nodulation stages (45 DAI) that an increased nodulation profile was verified. Also, the pRKACC-transformed strain showed similar nitrogen fixation abilities when compared to the wild-type strain. Subsequently, Nascimento et al. [81] showed that M. ciceri LMS-1 pRKACC maintained its increased nodulation abilities even when inoculated in different chickpea cultivars growing in soil. Using a similar approach, Brígido et al. [82] showed that a salt-sensitive Mesorhizobium strain was able to induce nodules in chickpea plants to the same extent as a salt-tolerant strain by expressing an exogenous acdS gene (pRKACC), further emphasizing the role of ACC deaminase in the nodulation abilities of these strains specially under environmental stress (salinity) conditions. Recently, Kong et al. [74] showed that S. meliloti CCNWSX0020 expressing the pRKACC plasmid presented augmented nodulation abilities in Medicago lupulina plants. Although S. meliloti CCNWSX0020 possesses a functional acdS gene in its symbiotic plasmid and contains a moderate level of ACC deaminase activity, the expression of an exogenous (highly active) ACC deaminase still increased its nodulation abilities. Moreover, plants (Medicago lupulina) treated with this engineered strain displayed improved plant growth as well as copper tolerance and enhanced an antioxidant defense system.

Altogether, these data suggest that modulation of the ethylene levels in root tissues through ACC deaminase is an effective strategy to increase nodulation and competitiveness of the bacterium, supporting the previous hypothesis suggested by $\mathrm{Ma}$ et al. [58], as well as to increase the ability to counteract the negative effects of environmental stresses.

Contrary to what has been observed in many rhizobial strains, an acdS insertion mutant of Bradyrhizobium japonicum did not show an altered nodulation phenotype in four different hosts; rather transcriptomic analysis showed that the acdS gene of Bradyrhizobium japonicum was upregulated under symbiotic conditions [71]. The authors suggest that ACC deaminase effects in nodulation might not be common to all rhizobia. However, this study raises a number of questions. For instance, Murset et al. [71] reported the obtention of an acdS insertion mutant, but ACC deaminase activity was detected under free-living conditions $(0.053 \mu \mathrm{mol} \alpha$ ketobutyrate/mg) and inside the nodules formed by the mutant strain ( 0.17 to $0.59 \mu \mathrm{mol} \alpha$-ketobutyrate/mg). On the other hand, Uchiumi et al. [70] have demonstrated that ACC deaminase activity of approximately $0.075 \mu \mathrm{mol} \alpha$ ketobutyrate/mg protein/h was sufficient to induce increased nodulation abilities in Mesorhizobium sp. MAFF303099. This suggests that, in the study reported by Murset et al. [71], the ACC deaminase activity in the mutant strain was sufficient to decrease the ethylene levels. Also, the presence of rhizobitoxine, which is present in many Bradyrhizobium japonicum strains [65], could decrease the ethylene levels even in the absence of ACC deaminase. The timing of the nodulation assays can also explain the absence of differences. For instance, Nascimento et al. [78] only observed differences in the nodulation profile between a wild-type and acdSoverproducing strain after 45 days after inoculation.

The overwhelming weight of evidence indicates that rhizobia expressing ACC deaminase naturally or through genetically engineering are more competitive and increase nodulation in legumes and consequently contribute to plant growth and development. This suggests that a relatively high level of ACC deaminase activity might be very important for developing rhizobial inocula with increased nodulation abilities even under environmental stress conditions. 


\section{Coinoculation of Legumes with Rhizobia and Other ACC Deaminase-Producing Bacteria}

Despite the low ACC deaminase activity in some rhizobial strains, it is generally sufficient to facilitate the nodulation process in the host plants but not to decrease the high levels of ethylene formed in plant roots due to various environmental stresses [26]. This fact becomes more important when legumes are grown in marginal soils, where the stress conditions may be a limiting factor for the establishment of a successful rhizobium-legume symbiosis. In this sense, a strategy to increase nodulation, especially under stress conditions, may rely on the use of a combination of both rhizobial strains and other ACC deaminase-producing bacteria. In fact, coinoculation of legumes with plant growthpromoting bacteria (PGPB) containing ACC deaminase and compatible rhizobia has proven to be a very useful approach for promoting nodulation by lowering ethylene concentrations in infected roots. For example, Shaharoona et al. [60] reported that coinoculation with a PGPB possessing ACC deaminase activity and $B$. japonicum resulted in up to $48 \%$ better nodulation in mung bean plants compared with single inoculation of B. japonicum. Similarly, Remans et al. [83] verified that coinoculation of PGPB along with rhizobia resulted in enhanced nodulation in common beans. Furthermore, inoculation of chickpea and lentil plants with a consortium of rhizobia and rhizospheric bacteria (with high ACC deaminase activity) resulted in increased nodulation and plant growth $[84,85]$. Not only did coinoculation of ACC deaminase-producing bacteria along with respective rhizobia contribute to a higher nodulation ability, but also it was also reported that by adjusting ethylene levels, an improvement of plant growth and yield was obtained in different plants even when grown under stress conditions. For example, coinoculation of plants with rhizobia and ACC deaminase-containing bacteria strains enhanced nodulation and plant growth [86], even under stress conditions [87]. Another study, conducted by Belimov et al. [62], showed that pea plants inoculated with an inocula containing Variovorax paradoxus 5C-2, carrying an ACC deaminase gene, and an appropriate rhizobia strain yielded a higher level of nodulation and prevented the negative effects of drought stress compared to the plants inoculated with an inocula containing the rhizobia and an ACC deaminase minus mutant of $V$. paradoxus 5C-2. Safronova et al. [80] demonstrated that the coinoculation of Mesorhizobium loti strains and $V$. paradoxus $5 \mathrm{C}-2$, both possessing ACC deaminase activity, had synergistic and additive effects on nodule number, root growth, and uptake of elements ( $\mathrm{N}, \mathrm{P}, \mathrm{Ca}, \mathrm{Mg}, \mathrm{Na}, \mathrm{Mn}, \mathrm{Zn}$, and $\mathrm{Pb}$ ) in shoots of L.edulis and L. ornithopodioides. A study performed by Ahmad et al. [88] observed that coinoculation of rhizobia and a strain of Pseudomonas containing ACC deaminase could be effective for reducing the deleterious effects of salinity on growth, physiology, and quality of mung bean. Another study performed by Tittabutr et al. [89] revealed that coinoculation of mung bean with Bradyrhizobium and a PGPB that contained a stress-induced ACC deaminase enzyme alleviates the effects of different environmental stresses. Similarly, the nodulation and growth of chickpea was increased by coinoculation of Mesorhizobium ciceri and a PGPB with ACC deaminase activity, under irrigated and rainfed conditions compared to inoculation with rhizobium alone [90].

Collectively, the data clearly indicate that PGPB that contain ACC deaminase can be coinoculated with rhizobia to improve the resistance of plants to environmental stresses by lowering the content of stress-induced ethylene in plants as well as increase the extent of nodulation of cognate legumes.

\section{Conclusions}

Rhizobia are an important component of sustainable agriculture due to their ability to fix nitrogen from atmosphere in association with legumes. However, this symbiotic rhizobium-legume association is dependent on the efficiency and competitiveness of the rhizobial strain for nodulation with indigenous soil bacteria and environmental factors. Ethylene is a phytohormone that negatively affects the nodulation process and its concentration increases significantly when plants are grown under unfavorable conditions. Thus, lowering the amount of ethylene synthesis in the nodulating roots could contribute to an improvement in legume nodulation. The rhizobial enzyme ACC deaminase is one of the mechanisms that confers a higher nodulation efficiency and competiveness ability to rhizobia and may also decrease the negative effects caused by various environmental stresses on the nodulation process. Therefore, the selection and use of rhizobial strains with high ACC deaminase activity is a promising strategy to improve the performance of rhizobia-legumes symbioses. A similar strategy consisting of the coinoculating legumes with the combination of specific PGPB with high ACC deaminase activity and compatible rhizobial strains is also likely to achieve a high level of nodulation, growth, and yield of the inoculated legumes, traits that are important for achieving an optimal/maximum symbiotic rhizobia-legume association.

\section{Competing Interests}

The authors declare that they have no competing interests.

\section{Acknowledgments}

Francisco X. Nascimento acknowledges a Ph.D. fellowship (SFRH/BD/86954/2012) from Fundação de Ciência e Tecnologia (FCT), Portugal. Clarisse Brígido acknowledges a FCT fellowship (SFRH/BPD/94751/2013).

\section{References}

[1] P. De Hoff and A. M. Hirsch, "Nitrogen comes down to earth: report from the 5th European Nitrogen Fixation Conference," Molecular Plant-Microbe Interactions, vol. 16, no. 5, pp. 371-375, 2003.

[2] D. J. Gage, "Infection and invasion of roots by symbiotic, nitrogen-fixing rhizobia during nodulation of temperate 
legumes," Microbiology and Molecular Biology Reviews, vol. 68, no. 2, pp. 280-300, 2004.

[3] G. E. D. Oldroyd and J. A. Downie, "Coordinating nodule morphogenesis with rhizobial infection in legumes," Annual Review of Plant Biology, vol. 59, pp. 519-546, 2008.

[4] J. A. Downie, "Legume nodulation," Current Biology, vol. 24, no. 5, pp. R184-R190, 2014.

[5] E. Limpens and T. Bisseling, "Signaling in symbiosis," Current Opinion in Plant Biology, vol. 6, no. 4, pp. 343-350, 2003.

[6] J. E. Cooper, "Early interactions between legumes and rhizobia: disclosing complexity in a molecular dialogue," Journal of Applied Microbiology, vol. 103, no. 5, pp. 1355-1365, 2007.

[7] U. B. Priefer, J. Aurag, B. Boesten et al., "Characterisation of Phaseolus symbionts isolated from Mediterranean soils and analysis of genetic factors related to $\mathrm{pH}$ tolerance," Journal of Biotechnology, vol. 91, no. 2-3, pp. 223-236, 2001.

[8] P. W. Singleton, S. A. El Swaify, and B. B. Bohlool, "Effect of salinity on Rhizobium growth and survival," Applied and Environmental Microbiology, vol. 44, no. 4, pp. 884-890, 1982.

[9] P. H. Graham, S. E. Viteri, F. Mackie, A. T. Vargas, and A. Palacios, "Variation in acid soil tolerance among strains of Rhizobium phaseoli," Field Crops Research, vol. 5, pp. 121-128, 1982.

[10] F. Zhang and D. L. Smith, "Genistein accumulation in soybean (Glycine max [L.] Merr.) root systems under suboptimal root zone temperatures," Journal of Experimental Botany, vol. 47, no. 299, pp. 785-792, 1996.

[11] A. M. Ibekwe, J. S. Angle, R. L. Chaney, and P. van Berkum, "Enumeration and $\mathrm{N}_{2}$ fixation potential of Rhizobium leguminosarum biovar trifolii grown in soil with varying $\mathrm{pH}$ values and heavy metal concentrations," Agriculture, Ecosystems \& Environment, vol. 61, no. 2-3, pp. 103-111, 1997.

[12] M. Hungria and G. Stacey, "Molecular signals exchanged between host plants and rhizobia: basic aspects and potential application in agriculture," Soil Biology and Biochemistry, vol. 29, no. 5-6, pp. 819-830, 1997.

[13] M. Hungria and M. A. T. Vargas, "Environmental factors affecting $\mathrm{N}_{2}$ fixation in grain legumes in the tropics, with an emphasis on Brazil," Field Crops Research, vol. 65, no. 2-3, pp. 151-164, 2000.

[14] Z. Zhengwei, W. Fang, H. Y. Lee, and Z. Yang, "Responses of Azorhizobium caulinodans to cadmium stress," FEMS Microbiology Ecology, vol. 54, no. 3, pp. 455-461, 2005.

[15] M. J. Soto, A. Domínguez-Ferreras, D. Pérez-Mendoza, J. Sanjuán, and J. Olivares, "Mutualism versus pathogenesis: the give-and-take in plant-bacteria interactions," Cellular Microbiology, vol. 11, no. 3, pp. 381-388, 2009.

[16] K.-I. Yuhashi, N. Ichikawa, H. Ezura et al., "Rhizobitoxine production by Bradyrhizobium elkanii enhances nodulation and competitiveness on Macroptilium atropurpureum," Applied and Environmental Microbiology, vol. 66, no. 6, pp. 2658-2663, 2000.

[17] M. Honma and T. Shimomura, "Metabolism of 1-aminocyclopropane-1-carboxylic," Agricultural and Biological Chemistry, vol. 42, no. 10, pp. 1825-1831, 1978.

[18] A. B. Bleecker and H. Kende, "Ethylene: a gaseous signal molecule in plants," Annual Review of Cell and Developmental Biology, vol. 16, pp. 1-18, 2000.

[19] Z. Lin, S. Zhong, and D. Grierson, "Recent advances in ethylene research," Journal of Experimental Botany, vol. 60, no. 12, pp. 3311-3336, 2009.
[20] A. K. Mattoo and J. C. Suttle, The Plant Hormone Ethylene, CRC Press, Boca Raton, Fla, USA, 1991.

[21] F. B. Abeles, P. W. Morgan, and M. E. Salveit, Ethylene in Plant Biology, Academic Press, San Diego, Calif, USA, 1992.

[22] L. C. van Loon and B. R. Glick, "Increased plant fitness by rhizobacteria," in Molecular Ecotoxicology of Plants, H. Sandermann, Ed., vol. 170 of Ecological Studies, pp. 177-205, Springer, Berlin, Germany, 2004.

[23] H. Hyodo, "Stress/wound ethylene," in The Plant Hormone Ethylene, A. K. Mattoo and J. C. Shuttle, Eds., pp. 65-80, CRC Press, Boca Raton, Fla, USA, 1991.

[24] B. R. Glick, B. Todorovic, J. Czarny, Z. Cheng, J. Duan, and B. McConkey, "Promotion of plant growth by bacterial ACC deaminase," Critical Reviews in Plant Sciences, vol. 26, no. 5-6, pp. 227-242, 2007.

[25] V. I. Safronova, V. V. Stepanok, G. L. Engqvist, Y. V. Alekseyev, and A. A. Belimov, "Root-associated bacteria containing 1aminocyclopropane-1-carboxylate deaminase improve growth and nutrient uptake by pea genotypes cultivated in cadmium supplemented soil," Biology and Fertility of Soils, vol. 42, no. 3, pp. 267-272, 2006.

[26] B. R. Glick, "Bacteria with ACC deaminase can promote plant growth and help to feed the world," Microbiological Research, vol. 169, no. 1, pp. 30-39, 2014.

[27] D. O. Adams and S. F. Yang, "Ethylene biosynthesis: Identification of 1-aminocyclopropane-1-carboxylic acid as an intermediate in the conversion of methionine to ethylene," Proceedings of the National Academy of Sciences, vol. 76, no. 1, pp. 170-174, 1979.

[28] S. F. Yang and N. E. Hoffman, "Ethylene biosynthesis and its regulation in higher plants," Annual Review of Plant Physiology, vol. 35, no. 1, pp. 155-189, 1984.

[29] C. J. He, M. C. Drew, and P. W. Morgan, "Induction of enzymes associated with lysigenous aerenchyma formation in roots of Zea mays during hypoxia or nitrogen starvation," Plant Physiology, vol. 105, no. 3, pp. 861-865, 1994.

[30] R. K. Sairam, D. Kumutha, K. Ezhilmathi, P. S. Deshmukh, and G. C. Srivastava, "Physiology and biochemistry of waterlogging tolerance in plants," Biologia Plantarum, vol. 52, no. 3, pp. 401412, 2008.

[31] S. Duodu, T. V. Bhuvaneswari, T. J. W. Stokkermans, and N. K. Peters, "A positive role for rhizobitoxine in Rhizobium-legume symbiosis," Molecular Plant-Microbe Interactions, vol. 12, no. 12, pp. 1082-1089, 1999.

[32] A. S. Nandwal, S. Kukreja, N. Kumar et al., "Plant water status, ethylene evolution, N2-fixing efficiency, antioxidant activity and lipid peroxidation in Cicer arietinum L. nodules as affected by short-term salinization and desalinization," Journal of Plant Physiology, vol. 164, no. 9, pp. 1161-1169, 2007.

[33] Y. Ding and G. E. D. Oldroyd, "Positioning the nodule, the hormone dictum," Plant Signaling and Behavior, vol. 4, no. 2, pp. 89-93, 2009.

[34] N. Grobbelaar, B. Clarke, and M. C. Hough, "The nodulation and nitrogen fixation of isolated roots of Phaseolus vulgaris $\mathrm{L}$. III. The effect of carbon dioxide and ethylene," Plant and Soil, vol. 35, no. 1, pp. 215-223, 1971.

[35] D. S. H. Drennan and C. Norton, "The effect of ethrel on nodulation in Pisum sativum L.," Plant and Soil, vol. 36, no. 1, pp. 53-57, 1972.

[36] G. Goodlass and K. A. Smith, "Effects of ethylene on root extension and nodulation of pea (Pisum sativum L.) and white 
clover (Trifolium repens L.)," Plant and Soil, vol. 51, no. 3, pp. 387-395, 1979.

[37] N. K. Peters and D. K. Crist-Estes, "Nodule formation is stimulated by the ethylene inhibitor aminoethoxyvinylglycine," Plant Physiology, vol. 91, no. 2, pp. 690-693, 1989.

[38] J. C. Fearn and T. A. Larue, "Ethylene inhibitors restore modulation to sym 5 mutants of Pisum sativum L. cv sparkle," Plant Physiology, vol. 96, no. 1, pp. 239-244, 1991.

[39] K. H. Lee and T. A. LaRue, "Exogenous ethylene inhibits nodulation of Pisum sativum L. cv sparkle," Plant Physiology, vol. 100, no. 4, pp. 1759-1763, 1992.

[40] S. M. Tamimi and M. P. Timko, "Effects of ethylene and inhibitors of ethylene synthesis and action on nodulation in common bean (Phaseolus vulgaris L.)," Plant and Soil, vol. 257, no. 1, pp. 125-131, 2003.

[41] N. Nukui, H. Ezura, K.-I. Yuhashi, T. Yasuta, and K. Minamisawa, "Effects of ethylene precursor and inhibitors for ethylene biosynthesis and perception on nodulation in Lotus japonicus and Macroptilium atropurpureum," Plant and Cell Physiology, vol. 41, no. 7, pp. 893-897, 2000.

[42] G. E. D. Oldroyd, E. M. Engstrom, and S. R. Long, "Ethylene inhibits the Nod factor signal transduction pathway of Medicago truncatula," Plant Cell, vol. 13, no. 8, pp. 1835-1849, 2001.

[43] R. V. Penmetsa and D. R. Cook, "A legume ethylene-insensitive mutant hyperinfected by its rhizobial symbiont," Science, vol. 275, no. 5299, pp. 527-530, 1997.

[44] R. V. Penmetsa, P. Uribe, J. Anderson et al., "The Medicago truncatula ortholog of Arabidopsis EIN2, sickle, is a negative regulator of symbiotic and pathogenic microbial associations," The Plant Journal, vol. 55, no. 4, pp. 580-595, 2008.

[45] K. Miyata, M. Kawaguchi, and T. Nakagawa, "Two distinct EIN2 genes cooperatively regulate ethylene signaling in Lotus japonicus," Plant and Cell Physiology, vol. 54, no. 9, pp. 14691477, 2013.

[46] N. Nukui, H. Ezura, and K. Minamisawa, "Transgenic Lotus japonicus with an ethylene receptor gene Cm-ERS1/H70A enhances formation of infection threads and nodule primordia," Plant and Cell Physiology, vol. 45, no. 4, pp. 427-435, 2004.

[47] D. Lohar, J. Stiller, J. Kam, G. Stacey, and P. M. Gresshoff, "Ethylene insensitivity conferred by a mutated arabidopsis ethylene receptor gene alters nodulation in transgenic Lotus japonicus," Annals of Botany, vol. 104, no. 2, pp. 277-285, 2009.

[48] B. J. Ferguson and U. Mathesius, "Phytohormone regulation of legume-rhizobia interactions," Journal of Chemical Ecology, vol. 40, no. 7, pp. 770-790, 2014.

[49] F. Csukasi, C. Merchante, and V. Valpuesta, "Modification of plant hormone levels and signaling as a tool in plant biotechnology," Biotechnology Journal, vol. 4, no. 9, pp. 12931304, 2009.

[50] B. Patrick, L. Antonin, L.-L. Servane, C. Deleu, and E. Le Deunff, "Ethylene modifies architecture of root system in response to stomatal opening and water allocation changes between root and shoot," Plant Signaling and Behavior, vol. 4, no. 1, pp. 4446, 2009.

[51] R. Heidstra, W. C. Yang, Y. Yalcin et al., "Ethylene provides positional information on cortical cell division but is not involved in Nod factor-induced root hair tip growth in Rhizobium-legume interaction," Development, vol. 124, no. 9, pp. 1781-1787, 1997.

[52] M. Sugawara, S. Okazaki, N. Nukui, H. Ezura, H. Mitsui, and K. Minamisawa, "Rhizobitoxine modulates plant-microbe interactions by ethylene inhibition," Biotechnology Advances, vol. 24, no. 4, pp. 382-388, 2006.
[53] P. K. Chan, B. Biswas, and P. M. Gresshoff, "Classical ethylene insensitive mutants of the Arabidopsis EIN2 orthologue lack the expected 'hypernodulation' response in lotus japonicus," Journal of Integrative Plant Biology, vol. 55, no. 4, pp. 395-408, 2013.

[54] S. Gonzalez-Rizzo, M. Crespi, and F. Frugier, "The Medicago truncatula CRE1 cytokinin receptor regulates lateral root development and early symbiotic interaction with Sinorhizobium meliloti," Plant Cell, vol. 18, no. 10, pp. 2680-2693, 2006.

[55] P. H. Middleton, J. Jakab, R. V. Penmetsa et al., "An ERF transcription factor in Medicago truncatula that is essential for nod factor signal transduction," Plant Cell, vol. 19, no. 4, pp. 1221-1234, 2007.

[56] F. C. Guinel and R. D. Geil, "A model for the development of the rhizobial and arbuscular mycorrhizal symbioses in legumes and its use to understand the roles of ethylene in the establishment of these two symbioses," Canadian Journal of Botany, vol. 80, no. 7, pp. 695-720, 2002.

[57] B. J. Ferguson, A. Indrasumunar, S. Hayashi et al., "Molecular analysis of legume nodule development and autoregulation," Journal of Integrative Plant Biology, vol. 52, no. 1, pp. 61-76, 2010.

[58] W. Ma, S. B. Sebestianova, J. Sebestian, G. I. Burd, F. C. Guinel, and B. R. Glick, "Prevalence of 1-aminocyclopropane1-carboxylate deaminase in Rhizobium spp," Antonie van Leeuwenhoek, vol. 83, no. 3, pp. 285-291, 2003.

[59] W. Ma, T. C. Charles, and B. R. Glick, "Expression of an exogenous 1-aminocyclopropane-1-carboxylate deaminase gene in Sinorhizobium meliloti increases its ability to nodulate alfalfa," Applied and Environmental Microbiology, vol. 70, no. 10, pp. 5891-5897, 2004.

[60] B. Shaharoona, M. Arshad, and Z. A. Zahir, "Effect of plant growth promoting rhizobacteria containing ACC-deaminase on maize (Zea mays L.) growth under axenic conditions and on nodulation in mung bean (Vigna radiata L.)," Letters in Applied Microbiology, vol. 42, no. 2, pp. 155-159, 2006.

[61] M. Saleem, M. Arshad, S. Hussain, and A. S. Bhatti, "Perspective of plant growth promoting rhizobacteria (PGPR) containing ACC deaminase in stress agriculture," Journal of Industrial Microbiology and Biotechnology, vol. 34, no. 10, pp. 635-648, 2007.

[62] A. A. Belimov, I. C. Dodd, N. Hontzeas, J. C. Theobald, V. I. Safronova, and W. J. Davies, "Rhizosphere bacteria containing 1aminocyclopropane-1-carboxylate deaminase increase yield of plants grown in drying soil via both local and systemic hormone signalling," New Phytologist, vol. 181, no. 2, pp. 413-423, 2009.

[63] M. A. Parker and N. K. Peters, "Rhizobitoxine production and symbiotic compatibility of Bradyrhizobium from Asian and North American lineages of Amphicarpaea," Canadian Journal of Microbiology, vol. 47, no. 10, pp. 889-894, 2001.

[64] X. A. Ruan and N. K. Peters, "Isolation and characterization of rhizobitoxine mutants of Bradyrhizobium japonicum," Journal of Bacteriology, vol. 174, no. 11, pp. 3467-3473, 1992.

[65] W. C. Ratcliff, S. V. Kadam, and R. F. Denison, "Poly-3hydroxybutyrate (PHB) supports survival and reproduction in starving rhizobia," FEMS Microbiology Ecology, vol. 65, no. 3, pp. 391-399, 2008.

[66] F. X. Nascimento, M. J. Rossi, C. R. F. S. Soares, B. J. McConkey, and B. R. Glick, "New insights into 1-aminocyclopropane-1carboxylate (ACC) deaminase phylogeny, evolution and ecological significance," PLoS ONE, vol. 9, Article ID e99168, 2014.

[67] W. Ma, F. C. Guinel, and B. R. Glick, "Rhizobium leguminosarum biovar viciae 1-aminocyclopropane-1-carboxylate deaminase 
promotes nodulation of pea plants," Applied and Environmental Microbiology, vol. 69, no. 8, pp. 4396-4402, 2003.

[68] J. Duan, K. M. Müller, T. C. Charles, S. Vesely, and B. R. Glick, "1Aminocyclopropane-1-carboxylate (ACC) deaminase genes in rhizobia from southern Saskatchewan," Microbial Ecology, vol. 57, no. 3, pp. 423-436, 2009.

[69] M. Stiens, S. Schneiker, M. Keller, S. Kuhn, A. Pühler, and A. Schlüter, "Sequence analysis of the 144-kilobase accessory plasmid pSmeSM11a, isolated from a dominant Sinorhizobium meliloti strain identified during a long-term field release experiment," Applied and Environmental Microbiology, vol. 72, no. 5, pp. 3662-3672, 2006.

[70] T. Uchiumi, T. Ohwada, M. Itakura et al., "Expression islands clustered on the symbiosis island of the Mesorhizobium loti genome," Journal of Bacteriology, vol. 186, no. 8, pp. 2439-2448, 2004.

[71] V. Murset, H. Hennecke, and G. Pessi, "Disparate role of rhizobial ACC deaminase in root-nodule symbioses," Symbiosis, vol. 57, no. 1, pp. 43-50, 2012.

[72] D. N. Fedorov, G. A. Ekimova, N. V. Doronina, and Y. A. Trotsenko, "1-Aminocyclopropane-1-carboxylate (ACC) deaminases from Methylobacterium radiotolerans and Methylobacterium nodulans with higher specificity for ACC," FEMS Microbiology Letters, vol. 343, no. 1, pp. 70-76, 2013.

[73] J. Onofre-Lemus, I. Hernández-Lucas, L. Girard, and J. Caballero-Mellado, "ACC (1-aminocyclopropane-1-carboxylate) deaminase activity, a widespread trait in Burkholderia species, and its growth-promoting effect on tomato plants," Applied and Environmental Microbiology, vol. 75, no. 20, pp. 6581-6590, 2009.

[74] Z. Kong, B. R. Glick, J. Duan et al., "Effects of 1-aminocyclopropane-1-carboxylate (ACC) deaminase-overproducing Sinorhizobium meliloti on plant growth and copper tolerance of Medicago lupulina," Plant and Soil, vol. 391, no. 1-2, pp. 383-398, 2015.

[75] B. R. Glick and J. C. Stearns, "Making phytoremediation work better: maximizing a plants growth potential in the midst of adversity," International Journal of Phytoremediation, vol. 13, pp. 4-16, 2011.

[76] F. X. Nascimento, C. Brígido, B. R. Glick, and S. Oliveira, "ACC deaminase genes are conserved among Mesorhizobium species able to nodulate the same host plant," FEMS Microbiology Letters, vol. 336, no. 1, pp. 26-37, 2012.

[77] N. Nukui, K. Minamisawa, S.-I. Ayabe, and T. Aoki, "Expression of the 1-aminocyclopropane-1-carboxylic acid deaminase gene requires symbiotic nitrogen-fixing regulator gene nifA2 in Mesorhizobium loti MAFF303099," Applied and Environmental Microbiology, vol. 72, no. 7, pp. 4964-4969, 2006.

[78] F. Nascimento, C. Brígido, L. Alho, B. R. Glick, and S. Oliveira, "Enhanced chickpea growth-promotion ability of a Mesorhizobium strain expressing an exogenous ACC deaminase gene," Plant and Soil, vol. 353, no. 1-2, pp. 221-230, 2012.

[79] V. P. Conforte, M. Echeverria, C. Sánchez, R. A. Ugalde, A. B. Menéndez, and V. C. Lepek, "Engineered ACC deaminaseexpressing free-living cells of Mesorhizobium loti show increased nodulation efficiency and competitiveness on Lotus spp," Journal of General and Applied Microbiology, vol. 56, no. 4, pp. 331-338, 2010.

[80] V. I. Safronova, G. Piluzza, N. Y. Zinovkina, A. K. Kimeklis, A. A. Belimov, and S. Bullitta, "Relationships between pasture legumes, rhizobacteria and nodule bacteria in heavy metal polluted mine waste of SW Sardinia," Symbiosis, vol. 58, no. 1-3, pp. 149-159, 2012.

[81] F. X. Nascimento, C. Brígido, B. R. Glick, S. Oliveira, and L. Alho, "Mesorhizobium ciceri LMS-1 expressing an exogenous 1aminocyclopropane-1-carboxylate (ACC) deaminase increases its nodulation abilities and chickpea plant resistance to soil constraints," Letters in Applied Microbiology, vol. 55, no. 1, pp. 15-21, 2012.

[82] C. Brígido, F. X. Nascimento, J. Duan, B. R. Glick, and S. Oliveira, "Expression of an exogenous 1-aminocyclopropane-1carboxylate deaminase gene in Mesorhizobium spp. reduces the negative effects of salt stress in chickpea," FEMS Microbiology Letters, vol. 349, no. 1, pp. 46-53, 2013.

[83] R. Remans, A. Croonenborghs, R. T. Gutierrez, J. Michiels, and J. Vanderleyden, "Effects of plant growth-promoting rhizobacteria on nodulation of Phaseolus vulgaris L. are dependent on plant P nutrition," European Journal of Plant Pathology, vol. 119, no. 3, pp. 341-351, 2007.

[84] S. M. Shahzad, A. Khalid, M. Arshad, J. Tahir, and T. Mahmood, "Improving nodulation, growth and yield of Cicer arietinum L. through bacterial ACC-deaminase induced changes in root architecture," European Journal of Soil Biology, vol. 46, no. 5, pp. 342-347, 2010.

[85] Z. A. Zahir, M. Zafar-ul-Hye, S. Sajjad, and M. Naveed, "Comparative effectiveness of Pseudomonas and Serratia sp. containing ACC-deaminase for co-inoculation with Rhizobium leguminosarum to improve growth, nodulation, and yield of lentil," Biology and Fertility of Soils, vol. 47, no. 4, pp. 457-465, 2011.

[86] R. Dey, K. K. Pal, D. M. Bhatt, and S. M. Chauhan, "Growth promotion and yield enhancement of peanut (Arachis hypogaea L.) by application of plant growth-promoting rhizobacteria," Microbiological Research, vol. 159, no. 4, pp. 371-394, 2004.

[87] M. Ahmad, Z. A. Zahir, H. N. Asghar, and M. Asghar, "Inducing salt tolerance in mung bean through coinoculation with rhizobia and plant-growthpromoting rhizobacteria containing 1aminocyclopropane-1-carboxylate deaminase," Canadian Journal of Microbiology, vol. 57, no. 7, pp. 578-589, 2011.

[88] M. Ahmad, Z. A. Zahir, M. Khalid, F. Nazli, and M. Arshad, "Efficacy of Rhizobium and Pseudomonas strains to improve physiology, ionic balance and quality of mung bean under saltaffected conditions on farmer's fields," Plant Physiology and Biochemistry, vol. 63, pp. 170-176, 2013.

[89] P. Tittabutr, P. Piromyou, A. Longtonglang, R. Noisa-Ngiam, N. Boonkerd, and N. Teaumroong, "Alleviation of the effect of environmental stresses using co-inoculation of mungbean by Bradyrhizobium and rhizobacteria containing stress-induced ACC deaminase enzyme," Soil Science and Plant Nutrition, vol. 59, no. 4, pp. 559-571, 2013.

[90] S. M. Shahzad, A. Khalid, M. S. Arif et al., "Co-inoculation integrated with P-enriched compost improved nodulation and growth of chickpea (Cicer arietinum L.) under irrigated and rainfed farming systems," Biology and Fertility of Soils, vol. 50, no. 1, pp. 1-12, 2014. 


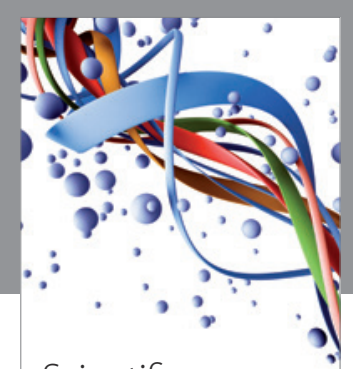

Scientifica
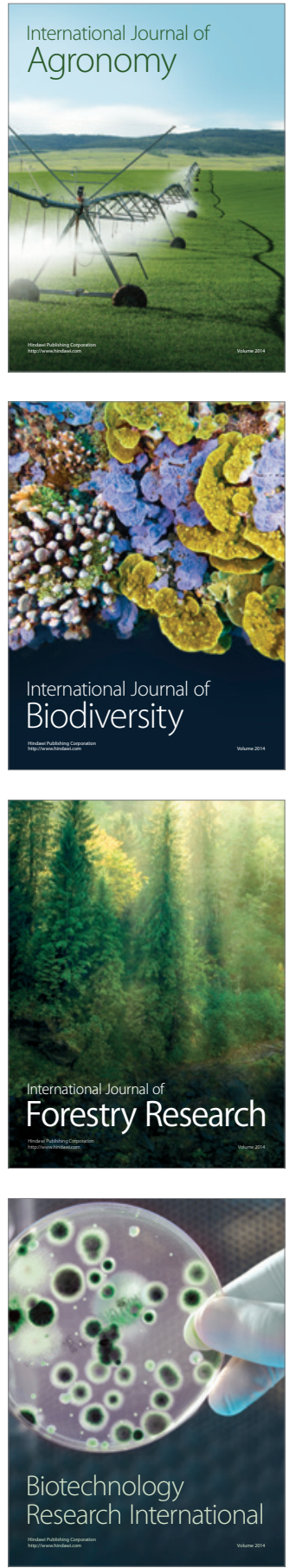
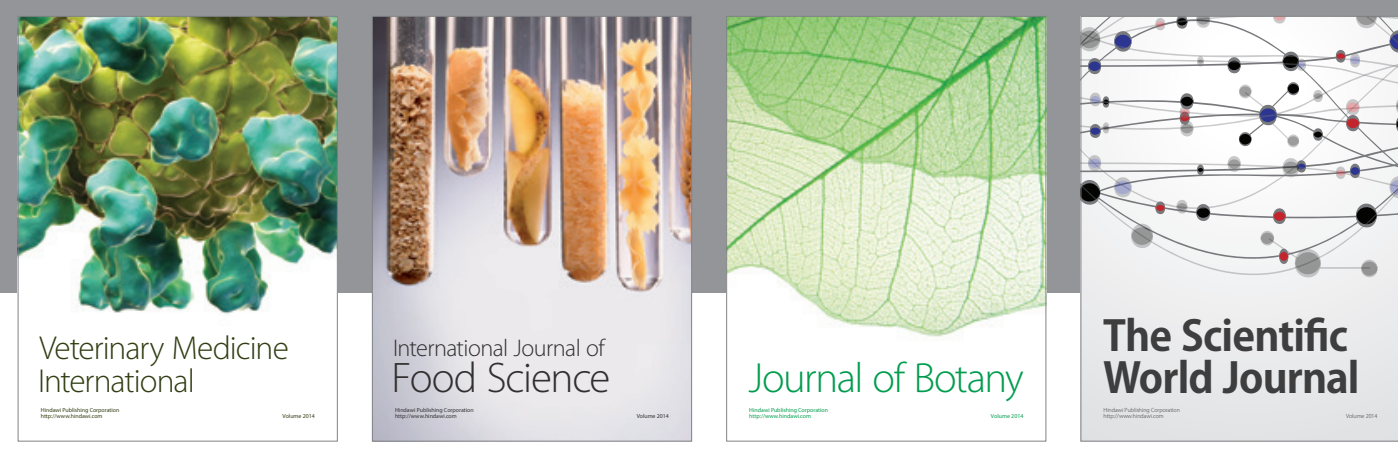

The Scientific

\section{World Journal}

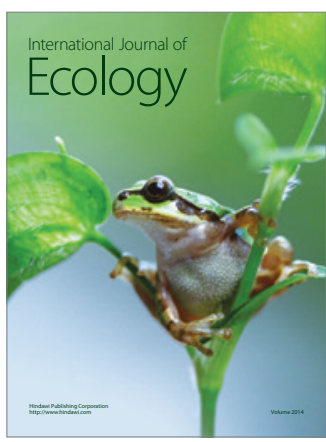

\section{Hindawi}

Submit your manuscripts at

http://www.hindawi.com
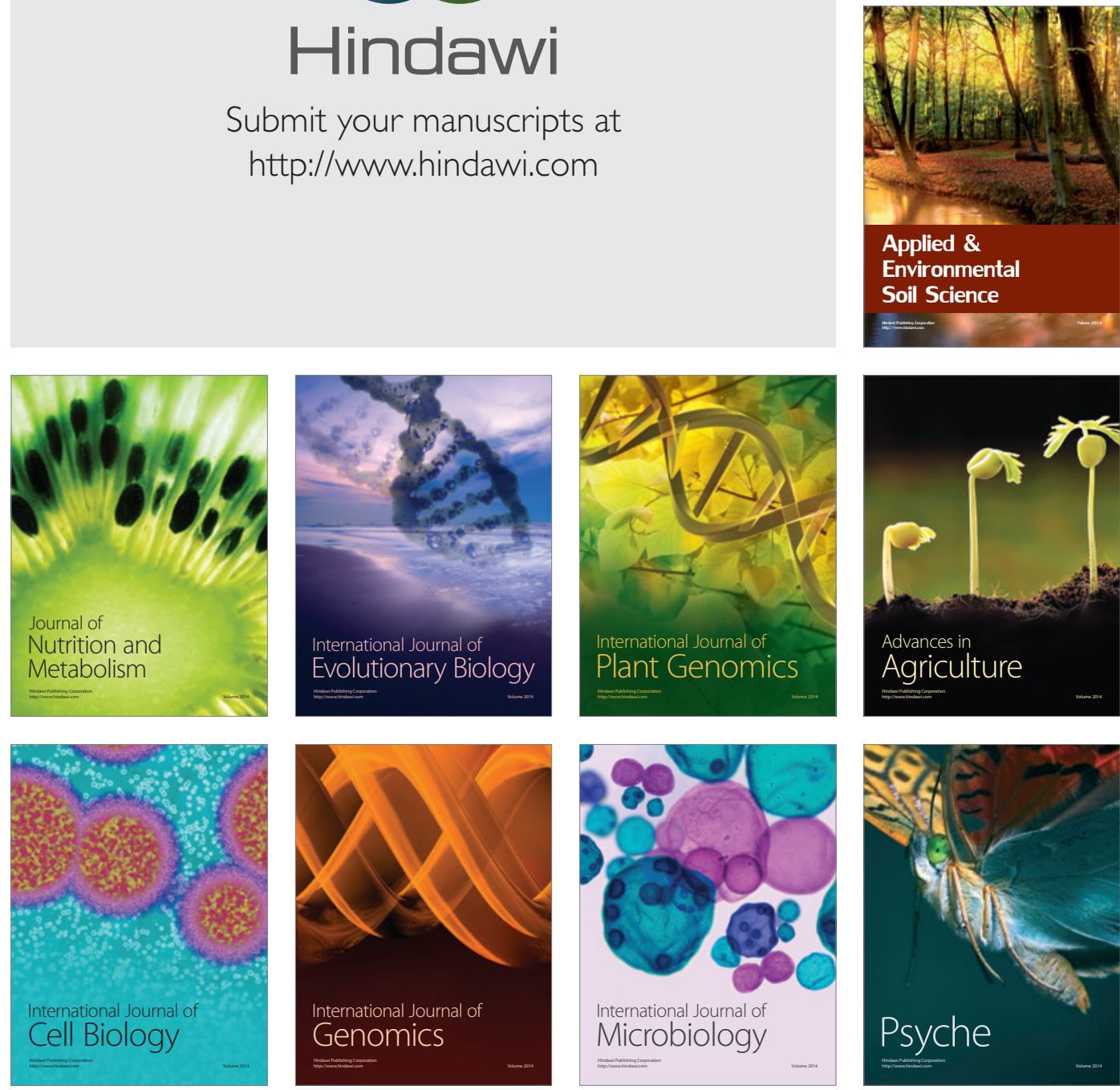
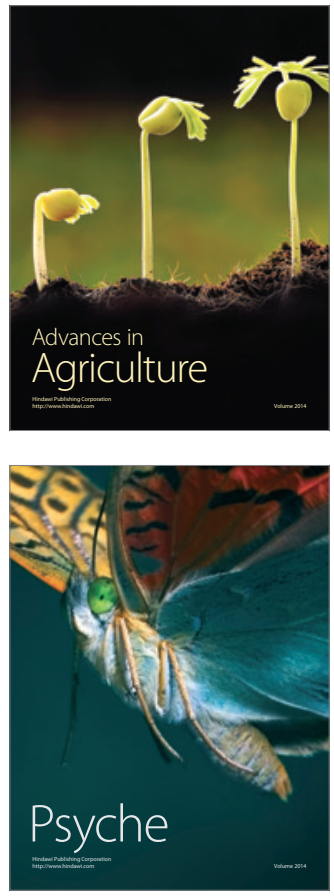